\title{
Ab in die Produktion oder Der diskrete Charme der Ökonomie
}

\author{
STEPHAN LESSENICH
}

Der Marsch in und durch die Institutionen war bei Revolutionären - bzw. bei jenen, die sich selbst als solche verstehen, - noch nie sonderlich beliebt. Wer in der Geschichte des gesellschaftlichen Fortschritts eine richtig große Nummer drehen wollte, der gab sich selten damit ab, das Neue im Alten zu suchen. Die Mühen der Ebene institutionellen Wandels sind die Sache sozialer Bewegungsprotagonisten nicht. Psychodynamisch erhebender und der revolutionären Selbstbeschreibung entsprechender scheint es zu sein, alte Zöpfe (früher gern auch gleich mal Köpfe) abschneiden zu wollen, um auf dem blanken Haupt des nackten Kaisers tausend neue Blumen erblühen zu lassen. Vergesst das Gestern und die ewig Gestrigen, mit uns kommt die neue Zeit: Sympathisch klingt das, nach frischem Wind und mitreißendem Elan, nach der Abkehr von eingefahrenen Pfaden und dem Aufbruch in ein neues, diesmal aber wirklich selbstbestimmtes Zeitalter. Bis zum nächsten Kommunenplenum und der Frage, wer den Dreck der anderen wegmacht.

\section{Irrtümer der Alternativproduktion}

Machen wir uns nichts vor: Wer die herrschenden Produktionsverhältnisse ändern will - ein Ziel, das hierzulande vielleicht wichtiger, in jedem Fall aber weltgesellschaftlich drängender ist als jemals zuvor -, der muss die herrschenden Produktionsverhältnisse ernstnehmen. Wer die herrschenden Verhältnisse der gesellschaftlichen Produktion und der produzierenden Herrschaft des Menschen über die „Natur“ wirklich grundlegend verändern will, der muss an die in diesen herrschenden gesellschaftlichen Produktions- und Naturverhältnissen arbeitenden Produzenten und Produzentinnen ran; an diejenigen, die - wie gut oder schlecht sie davon leben, wie bequem oder unbequem sie sich darin eingerichtet haben mögen - in diesen Verhältnissen existenziell gefangen, die vom gesellschaftlich Herrschenden befangen sind. Es geht um jene, die nicht über die Kraft und die Ressourcen, den Übermut und die
Unbekümmertheit verfügen, einfach mal aus diesen Verhältnissen auszubrechen - also der Welt des Kapitals und der Entfremdung, der betrieblichen Herrschaft und des Normalarbeitsverhältnisses den Rücken zu kehren und auf eigene Faust anders zu produzieren und zu konsumieren, $\mathrm{zu}$ arbeiten und zu leben. Und wenn's schiefgeht, dann gibt es ja noch das Ersparte der Eltern oder die Restbestände des versorgenden Sozialstaats.

Sich die eigenen, partikularen „Produktionsverhältnisse“ zu schaffen, ist schön und gut, aber unter den gegebenen ökonomischen Bedingungen ein gesellschaftliches Minderheitenprogramm. Gemeinsam, hier und heute, die soziale Gegenwelt einer autonomen Produktionsstruktur und einer lokalen Solidaritätskultur zu realisieren, ist ohne jeden Zweifel aller Ehren wert, ein Akt gelebten Eigensinns für die involvierten Akteure, ein sichtbares Zeichen alternativer Lebensführung. Aber es ist als solches nicht schon ein Schritt auf dem Weg in eine „andere Gesellschaft", kein kraft lebensphilosophischer Aufladung oder sozialwissenschaftlicher Begleitforschung verallgemeinerungsfähiges gesellschaftliches Veränderungsprojekt. Derartige Initiativen der Alternativproduktion sind nach Lage der Dinge keine Vorboten einer gesamtgesellschaftlichen Produktionsalternative - sie sind, um die Dinge und deren Lage dann doch einmal bei ihrem Namen zu nennen, Inseln (im besten Fall) nicht-kapitalistischer Organisationsformen im Meer der kapitalistischen Produktionsweise. Nicht weniger, aber bei Lichte besehen - und man mag das mit Fug und Recht bedauern - auch nicht mehr.

Es ist ja nicht nur so, dass die Vorstellung der Kollektivselbstständigkeit in basisdemokratisch regulierten Produktionsstrukturen nicht unbedingt jedermanns Sache auf dem Weg in einen anderen ökonomischen Vergesellschaftungsmodus sein dürfte. Es ist so, dass der Kapitalismus ein gesellschaftliches Herrschaftsverhältnis etabliert, aus dem nicht jeder und jede gleichermaßen individuell oder gemeinschaftlich ,auszubrechen“ vermag: Was dem nach einem antikapitalistischen
Erweckungserlebnis auf natureinträchtiges kleinunternehmerisches Slow Food-Cooking im ewigen Frühling der Kanaren umgestiegenen ExFinanzmanager möglich ist, bleibt der um ihren befristeten Niedriglohnarbeitsplatz im entwürdigenden Betriebsklima eines Auslieferungszentrums in Bad Hersfeld bangenden Schichtarbeiterin vermutlich - und vermutlich nicht nur befristet, sondern auf Dauer - verwehrt. Und es ist vor allen Dingen so, dass der Kapitalismus ein gesellschaftliches Produktionsverhältnis ist, von dem sich „unabhängig“ zu machen systemisch nur einer begrenzten Zahl von Wirtschaftssubjekten erlaubt ist - die in diesem gesellschaftlichen Rahmen selbstverständlich an die kapitalistischen Produktionsstrukturen und Zirkulationsprozesse angekoppelt sind und von ihrem extern-internen Sonderstatus, ihrem ökonomischen „Draußen im Drinnen“, leben. Nicht-kapitalistische Zellen führen eine - nicht normativ zu entwertende, rein analytisch zu verstehende - parasitäre Existenz im Gewebe des Kapitalismus. Sie sind nicht Stachel im, sondern Fleisch vom Fleische der kapitalistischen Produktionsweise.

\section{Anforderungen und Akteure gesell- schaftlichen Wandels}

Wer die kapitalistischen Produktionsverhältnisse umgestalten, ja umbrechen möchte - zum Schutz der natürlichen Restressourcen der Welt und zum Wohle der arbeitenden Menschen auf ihr -, der muss sich auch tatsächlich der Umgestaltung, dem Umbruch der kapitalistischen Produktionsverhältnisse widmen, der muss, statt in die Reservate der Paradiesvögel, in die Höhle des Löwen gehen: in die kapitalistische Produktion. Für diese Aufgabe ist in der funktional differenzierten, arbeitsteiligen Gesellschaft sogar eine Planstelle vorgesehen; sie wird traditionell von den Gewerkschaften eingenommen. Sie sind es, zu deren funktionaler Selbstbeschreibung und normativem Selbstverständnis es gehört, die Produktionsverhältnisse zu ändern, sprich: die Verhältnisse zu verschieben, 
unter denen gesellschaftliche Produktionsentscheidungen stattfinden und unter denen gesellschaftliches Arbeitsvermögen verausgabt wird. Es geht darum, diese Bedingungen zu verbessern: Schritt für Schritt zugunsten der unmittelbaren Produzentinnen und Produzenten, der lebendigen Arbeit und ihrer vitalen Interessen.

Auch wenn historisch nicht durchgängig offensichtlich sein sollte, ob gewerkschaftliche Organisation immer und überall diesem Ziel gerecht wird, auch wenn man an der Rationalität und Konsequenz ihrer Zielverfolgung ab und an zweifeln mag: Eine grundlegende, emanzipatorische postwachstumsgesellschaftliche Transformation der Produktionsverhältnisse wird - wenn überhaupt - nicht allein von ,außen“, sondern nur in Verbindung mit einer Dynamik von „innen“, aus den Binnenwelten der kapitalistischen Ökonomie heraus erfolgen. Und das heißt: Ohne die Gewerkschaften, ohne den politischen Willen und die organisatorische Macht der unter den gegebenen - kapitalistischen - Bedingungen vergesellschafteten Produzentinnen und Produzenten wird es nicht gehen. Eine nachhaltige Umgestaltung der Wachstumsgesellschaft, das so dringend nötige Neue, wird nicht ohne die Akteure der Wachstumsgesellschaft selbst, ohne die - ob nun mit oder gegen deren Willen - Trägergruppen des Alten zustande kommen. Wer das nicht sieht, ist nur bedingt politikfähig.

Aber wie kann über das Alte, Bestehende zugleich das Neue, das Revolutionierende in die Welt kommen? Hier ist vor allem eines gefragt: die kollektive Arbeit am gesellschaftlichen Imaginären. Die unbehagliche Ahnung, dass die bestehenden Produktionsverhältnisse - mit all ihren zerstörerischen Konsequenzen, mit ihren himmelschreienden globalen Ungleichheitseffekten - im wahrsten Sinne des Wortes „not sustainable“, also letztlich nicht aufrechtzuerhalten sind, wächst ja nicht nur in den avantgardistischen Protagonisten alltagsrevolutionärer Lebensformen. Sie verbreitet sich allmählich, wie ein schleichender gesellschaftlicher Klimawandel, auch bei „ganz normalen“ Menschen in „ganz normalen" - kapitalistischen - Arbeitsverhältnissen. Ihren Zweifeln die nur allzu berechtigte Nahrung zu geben, sie emanzipatorisch zu wenden und unter den gegebenen Produktionsverhältnissen zu organisieren: das ist die wichtigste $\mathrm{He}$ rausforderung, vor der die „Partei der Arbeit“ heute steht. Und Herausforderungen sind in unserem neoliberalen Zeitalter ja bekanntlich dazu da, angenommen zu werden.

\section{AUTOR}

STEPHAN LESSENICH, Dr. habil., ist Professor für Soziologie an der Ludwig-Maximilians-Universität München und Ko-Direktor der DFG-Kollegforschergruppe „Postwachstumsgesellschaften“ an der Friedrich-Schiller-Universität Jena. Arbeitsschwerpunkte: Vergleichende Wohlfahrtsstaatsforschung, Politische Soziologie gesellschaftlicherTransformationen.

stephan.lessenich@soziologie.uni-muenchen.de 